\title{
Gerenciamento de resíduos sólidos urbanos: cenários, custos e oportunidades
}

\section{Management of municipal solid waste: scenarios, costs and opportunities}

Data de entrada: $21 / 03 / 2018$

- Data de aprovação: 28/03/2019

Ritielli Berticelli' | Adalberto Pandolfo ${ }^{2}$ Eduardo Pavan Korf ${ }^{3}$ Pâmela Bia Pasquali² | Jéssica Mulinari ${ }^{4}$

DOI: https://doi.org/10.36659/dae.2020.063

\section{ORCID ID}

Berticelli R (D) https://orcid.org/0000-0003-4264-822X

Pandolfo A (D) https://orcid.org/0000-0001-6522-3481
KorfEP (DD https://orcid.org/0000-0003-2041-0173 Pasquali PB (D) https://orcid.org/0000-0001-9898-6346

Mulinari, J (D) https://orcid.org/0000-0003-1849-7062

\section{Resumo}

Objetivou-se avaliar a viabilidade econômica de cenários voltados para o gerenciamento dos resíduos sólidos urbanos (RSU), verificando se é possível reduzir os custos por meio do cumprimento da legislação vigente. A metodologia consistiu na criação de cenários com alternativas de gerenciamento de RSU para o município de Passo Fundo, localizado no sul do Brasil, com característica de médio porte. Foram criados dois cenários, o cenário inercial, que considerou a manutenção das condições atuais do sistema, e o cenário normativo, que propõe a criação de um novo modelo baseado nos preceitos da política nacional de resíduos sólidos, de acordo com a Lei Federal 12.305/2010. As principais características técnicas, econômicas e operacionais desses cenários foram levantadas e foi realizada a análise de viabilidade econômica dos mesmos. 0 resultado da pesquisa demonstrou que o cenário normativo é mais viável economicamente, reduzindo os custos com o gerenciamento dos RSU em aproximadamente $25 \%$. A pesquisa permitiu avaliar a estimativa de custos necessários para cada cenário e a sua conclusão retrata que é possível seguir os objetivos estabelecidos pela Política Nacional de Resíduos Sólidos e ao mesmo tempo reduzir os custos com a gestão dos RSU.

Palavras-chave: Gerenciamento de resíduos sólidos. Viabilidade econômica. Política Nacional de Resíduos Sólidos.

\section{Abstract}

This study evaluated the economic viability of municipal solid waste (MSW) management scenarios, verifying if it is possible to reduce costs through the compliance with current legislation. The methodology consisted in the creation of scenarios with MSW management alternatives for the city of Passo Fundo, located in southern Brazil, with mid-size feature. Two scenarios were created: the inertial scenario that considered the maintenance of the current system conditions and the normative scenario that proposed the creation of a model based on the principles of the National Solid Waste Policy. The main technical, economic and operational characteristics of these scenarios were gathered. The survey results showed that the normative scenario is more economically viable, reducing costs of MSW management by approximately $25 \%$. It was concluded that it is possible to follow the objectives set by the National Solid Waste Policy and, at the same time, to reduce costs of MSW management.

Keywords: Solid waste management. Economic viability. National Solid Waste Policy. Municipal Policies.

\footnotetext{
${ }^{1}$ Universidade de Cruz Alta - Cruz Alta - Rio Grande do Sul - Brasil.

${ }^{2}$ Universidade de Passo Fundo - Passo Fundo - Rio Grande do Sul - Brasil.

${ }^{3}$ Universidade Federal da Fronteira Sul - Erechim - Rio Grande do Sul - Brasil.

${ }^{4}$ Universidade Federal de Santa Catarina - Florianópolis - Santa Catarina - Brasil.

* Autora correspondente: ritiberticelliayahoo.com.br.
} 


\section{INTRODUÇÃO}

O aumento dos níveis de população, economia em expansão, a rápida urbanização e o melhora dos padrões de vida da comunidade aceleraram a taxa de geração de Resíduos Sólidos Urbanos RSU (MINGHUA et al., 2009).

Os municípios, responsáveis pela gestão de RSU, têm o desafio de proporcionar um sistema eficaz e eficiente para os habitantes (GUERRERO; MAAS; HOGLAND, 2013). No entanto, muitas vezes enfrentam dificuldades que vão além da capacidade da autoridade municipal de resolver (SUJAUDDIN; HUDA; RAFIQUL HOQUE, 2008), principalmente devido à falta de organização e de recursos financeiros e à complexidade do sistema (BURNLEY, 2007).

A gestão de RSU muitas vezes representa uma significativa proporção do orçamento municipal total recorrente em cidades de renda média ou baixa (SCHEINBERG; WILSON; RODIC, 2010). As parcerias público-privadas por meio da terceirização dos serviços surgiram como uma alternativa para melhorar o desempenho dos serviços a custos mais baixos (COINTREAU; GOPALAN; COAD, 2000; ZHU et al., 2007; ABDRABO, 2008). Porém a abordagem dos aspectos financeiros, como contabilidade de custos e avaliação financeira, é fundamental para garantir a sustentabilidade do sistema de gestão de resíduos sólidos, garantindo um serviço de qualidade com custos compatíveis e com constantes investimentos no setor (LOHRI; CAMENZIND; ZURBRÜGG, 2014). Esses métodos são raramente utilizados, e muitas vezes o município desconhece o custo real da prestação do serviço (BARTONE; BERNSTEIN; WRIGHT, 1990; DIAZ, 1999; SCHÜBELER, 1996; WILSON et al., 2012).

Sendo assim, antes de o poder público tomar qualquer decisão sobre como proceder como novas estratégias de gestão de RSU, é indispensável estabelecer um entendimento completo dos custos atuais para provisão dos serviços futuros e das respectivas receitas (HOORNWEG; LAM; CHAUDHRY, 2005). A falta de acompanhamento financeiro específico e análise de dados é uma das principais barreiras para não ser capaz de sustentar qualquer melhoria prevista do sistema de gestão dos RSU (HANRAHAN; SRIVASTAVA; RAMAKRISHNA, 2006; ZURBRÜGG; BECKER; VOEGELI, 2007; PARTHAN et al., 2012).

Alguns pesquisadores descrevem a importância de um quadro jurídico adequado e o quanto o mesmo contribui positivamente para o desenvolvimento de um sistema integrado de gestão de resíduos (ASASE et al., 2009), enquanto a ausência de condições satisfatórias (MRAYYAN; HAMDI, 2006) e regulamentos fracos (SENG et al., 2010) são prejudiciais.

A gestão integrada de resíduos sólidos é representada por um conjunto de princípios de gerenciamento ambiental e economicamente adequados, de forma sustentável e socialmente aceitável (TCHOBANOGLOUS; KREITH, 2002; McDOUGALL et al., 2001; WHITE; FRANKE; HINDLE, 1999). Devido ao foco na flexibilidade e especificidades às condições locais, a gestão integrada de resíduos não prescreve soluções; em vez disso, mantém princípios, sendo que cada região deve desenvolver sistemas próprios em respostas aos seus contextos (McDOUGALL et al., 2001).

No Brasil, a gestão dos RSU tem carecido de atenção necessária do poder público. 0 crescimento das cidades brasileiras não foi acompanhado pela provisão de infraestrutura e de serviços urbanos (MINISTÉRIO DO MEIO AMBIENTE, 2011). Em 2010 foi criada a Politica Nacional de Resíduos Sólidos, em que o cumprimento das diretrizes estabelecidas pela Lei Federal $\mathrm{n}^{\circ} 12.305$ (BRASIL, 2010) tornou-se obrigatório. Porém a maioria dos munícipios ainda não dispõe de alternativas para disposição final de rejeitos e acaba destinando-o a lixões ou terceirizando o serviço. 
Constatando os impactos que os resíduos sólidos podem causar e a precariedade da situação atual em vários municípios brasileiros, alternativas se impõem visando melhorar a gestão, aumentando sua eficiência, diminuindo seus custos e colaborando com a proteção do meio ambiente. Esta pesquisa justifica-se pela necessidade de buscar novos modelos de gestão integrada de resíduos sólidos urbanos, firmando um marco norteador para a consecução de uma gestão sustentável de municípios de médio porte.

Neste sentido, objetivou-se avaliar a viabilidade econômica de cenários voltados para o gerenciamento dos RSU de um município de médio porte, verificando se é possível reduzir os custos por meio do estabelecimento de uma nova dinâmica às políticas municipais, propondo uma atuação integrada entre os serviços prestados à popula- ção, visando maior eficiência, eficácia, integração social e sustentabilidade.

\subsection{Gestão Integrada de Resíduos Sólidos}

A Gestão Integrada de Resíduos Sólidos (GIRSU) é definida pela PNRS como um conjunto de ações voltadas para a busca de soluções para os resíduos sólidos, de forma a considerar as dimensões política, econômica, ambiental, cultural e social, com controle social e sob a premissa do desenvolvimento sustentável (BRASIL, 2010).

O conceito de GIRSU procura equilibrar três dimensões: eficácia ambiental, aceitabilidade social e acessibilidade econômica, conforme apresentado na Fig. 1 (McDOUGALL et al., 2001; MORRISSEY; BROWNE, 2004; MARSHALL; FARAHBAKHSH, 2013).

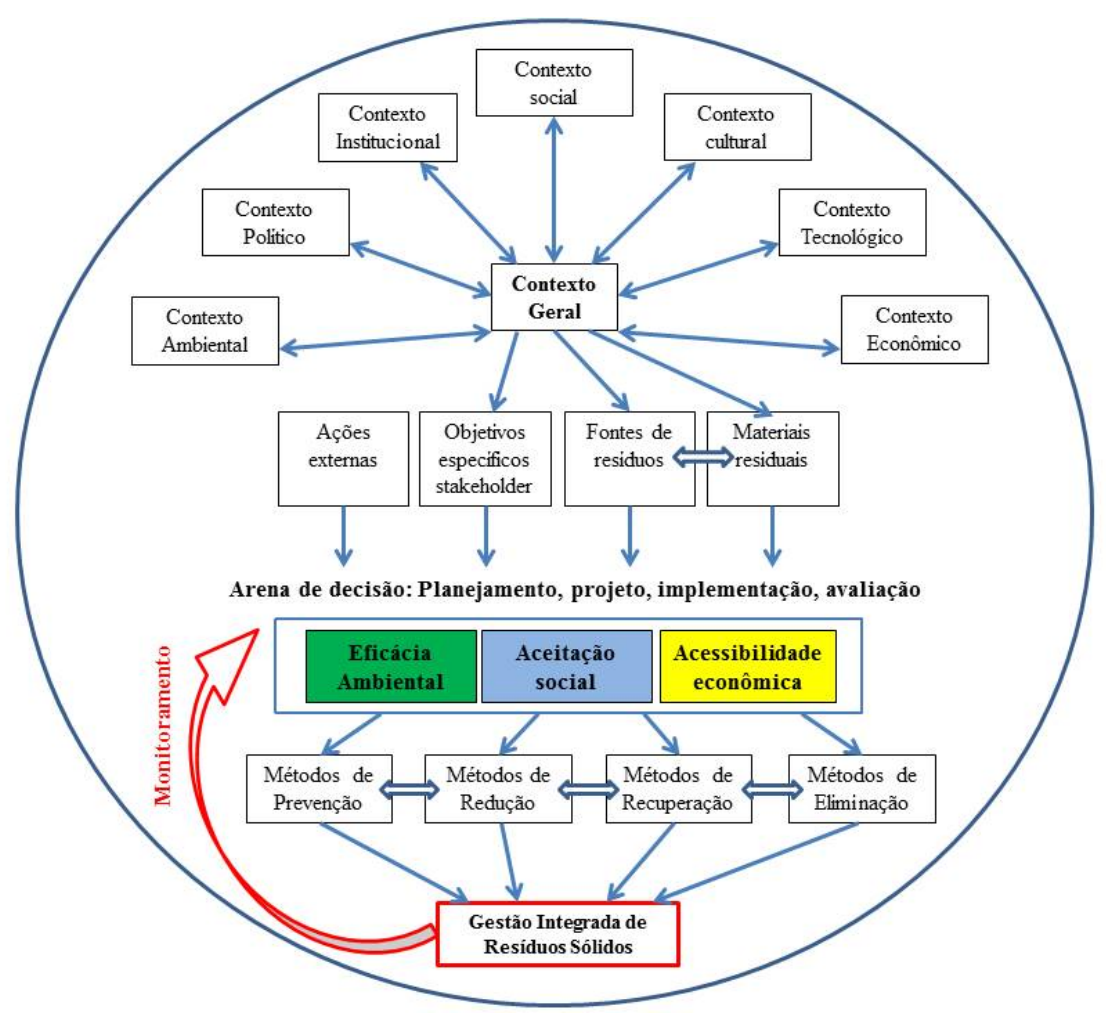

Figura 1 - Paradigma da Gestão Integrada de Resíduos Sólidos Fonte: Adaptado de Marshall e Farahbakhsh (2013) 
Para reduzir os impactos ambientais e diminuir os custos, um sistema deve ser integrado (em materiais de desperdício, fontes de resíduos e métodos de tratamento), orientado para o mercado (ou seja, energia e materiais têm usos finais) e flexível, permitindo a melhoria contínua (McDOUGALL et al., 2001).

Portanto, a gestão dos resíduos sólidos é uma tarefa complexa e abrangente, refletindo na dificuldade da maioria dos municípios, devido principalmente à falta de autonomia, recursos e conhecimento técnico sobre o assunto.

A Política Nacional de Resíduos Sólidos (PNRS) diferencia ainda os termos "gestão" e "gerenciamento". O gerenciamento de resíduos sólidos é o conjunto de ações exercidas nas etapas de coleta, transporte, transbordo, tratamento e destinação final ambientalmente adequada dos resíduos sólidos e disposição final ambientalmente adequada dos rejeitos, de acordo com os planos estabelecidos. Ou seja, "gerenciamento" é o termo a ser utilizado ao se tratar da logística do manejo de resíduos urbanos (BRASIL, 2010).

Muitas cidades ao redor do mundo estão buscando alternativas para o gerenciamento de RSU. A União Europeia (EU), por exemplo, tem se destacado por uma mudança significativa no modelo de gestão de RSU. O aproveitamento dos resíduos sólidos é o aspecto dominante. Em 2010, foram recuperados $40 \%$ dos resíduos descartados pela sociedade na forma de reciclagem e compostagem, $22 \%$ foram incinerados e 38\% dispostos em aterros sanitários. Vários países estão muito avançados quanto à redução de resíduos biodegradáveis em aterros sanitários; por exemplo, Suíça, Alemanha, Holanda, Suécia, Bélgica, Áustria e Dinamarca têm relatado taxas de aterramento desses resíduos abaixo de 5\% (JUCÁ et al., 2014).

Segundo Lopes (2003), a realidade brasileira quanto à gestão dos resíduos sólidos ainda se concentra na destinação final e não na prevenção da poluição e na minimização da geração de resíduos na fonte. A responsabilidade pela prestação de serviços de limpeza urbana é do município, e na maioria das cidades brasileiras a coleta de resíduos é realizada pela iniciativa privada sob forma de concessão, subcontratação ou permissão, onde ocorre a delegação de prestação dos serviços para pessoa física, jurídica ou consórcio, que demonstre capacidade para seu desempenho, por sua conta e risco. Como por exemplo, segundo Sistema Nacional de Informações sobre Saneamento (SNIS), no ano de $2016,50,16 \%$ da massa de RSU foi coletada por empresas contratadas pelas prefeituras por meio de terceirização. Ou seja, as empresas contratadas se constituem no maior executor da coleta seletiva em termos de massa, recoIhendo praticamente a metade da quantidade apurada pelo SNIS segundo as informações dos municípios (MINISTÉRIO DAS CIDADES, 2018).

A diversidade de cidades acarreta na geração de diferentes composições de resíduos, apontando para a necessidade de um contexto específico de gestão de resíduos, de acordo com cada região. Muitas tentativas de importar soluções de resíduos de países industrializados para países menos industrializados falharam porque os estudos indicam que os sistemas de gestão de resíduos do mundo em desenvolvimento foram cópias incompletas de um sistema ideal que opera em países desenvolvidos (UN-HABITAT, 2010). As cidades estão reconhecendo a necessidade de adaptação, soluções de gerenciamento de resíduos locais, sustentáveis que levam ao contexto local como um ponto de partida, não de uma tecnologia importada que talvez venha a não dar certo.

Rodrigo e Castells (2001) realizaram um estudo para elaboração de estratégias para o gerenciamento dos resíduos sólidos do município de Catalunha na Espanha e concluíram que os as- 
pectos econômicos e sociais também devem ser considerados para uma gestão eficiente.

O gerenciamento ambiental deve ser uma abordagem multilateral, considerando que os problemas ambientais e suas soluções são determinados por fatores tecnológicos, bem como por questões econômicas, sociais, físicas, culturais e políticas. Sendo assim, o sistema proposto é integrado, visando propostas sustentáveis em todos os setores (SHEN, 1995). Além disso, cabe salientar que enfoques diferenciados (cenários diferentes) podem alterar de forma significativa a capacidade e os custos do gerenciamento dos RSU, aumentando a sua capacidade e sustentabilidade. Desse modo, diferentes alternativas poderão subsidiar a tomada de decisão, favorecendo para que os municípios optem por sistemas mais sustentáveis.

\section{METODOLOGIA DA PESQUISA}

Este estudo apresenta modelos de gerenciamento de RSU que consideram um conjunto de tecnologias representativas de tratamento e disposição final de resíduos, que podem poten- cialmente ser implementados em municípios de médio porte no Brasil. Entende-se por município de médio porte aquele que possui de 100.000 a 500.000 habitantes.

A pesquisa foi realizada no município de Passo Fundo/RS. O município está localizado no estado do Rio Grande do Sul, pertencendo à Mesorregião Noroeste Rio-grandense (FAMURS, 2014). Possui área total de $780,36 \mathrm{~km}^{2}$ e uma população estimada para 2015 de 193.055 habitantes (PASSO FUNDO, 2014).

A Fig. 2 apresenta o fluxograma contendo as etapas da pesquisa. A etapa 1 foi elaborada considerando a realidade local do município estudado. Por meio de um estudo anterior e diagnóstico de muitas situações semelhantes em vários municípios brasileiros, foram criados dois cenários com a finalidade de identificar soluções integradas para o gerenciamento dos RSU. Um horizonte temporal de 20 anos foi adotado e cada cenário foi estudado individualmente, levantando-se as principais características técnicas e econômicas para a implantação, a instalação e a operacionalização dos cenários no município de estudo.
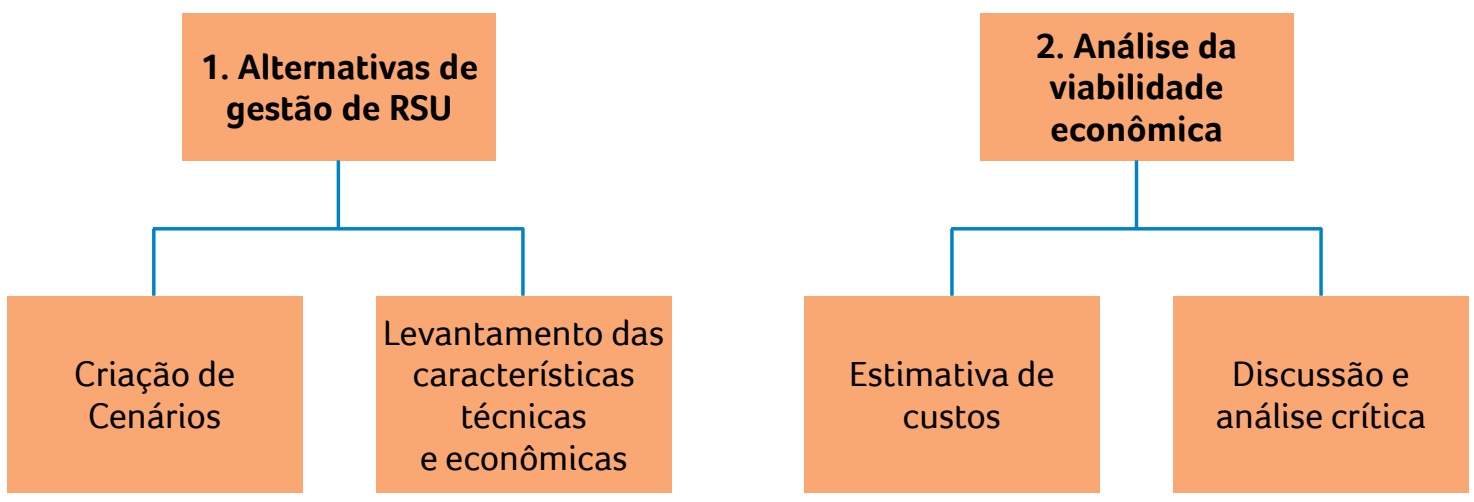

Figura 2 - Estrutura metodológica para desenvolvimento da pesquisa. 
O processo de geração de cenários englobou os temas de educação e sensibilização ambiental, coleta seletiva, reciclagem, aproveitamento da parcela orgânica dos resíduos sólidos urbanos e disposição final dos rejeitos. A criação dos cenários foi realizada com base na adaptação da metodologia descrita por Buarque (2003), onde é possível distinguir dois conjuntos diferenciados, em que a tendência do passado é mantida ou quando houver desenvolvimento ao longo do período de planejamento: cenários tendenciais e cenários normativos.

Por meio do estudo aprofundado foi possível propor cenários realísticos para o município e re- presentativo de municípios de médio porte. Poderia ser avaliado um cenário que considerasse a incineração, porém essa não é uma tendência aplicável em nível nacional. A incineração é pouco utilizada no Brasil, e os custos de implantação e operação ainda são bem elevados. Portanto, os cenários criados representam alternativas para o gerenciamento dos RSU, e a maior preocupação na criação dos cenários foi apresentar e comparar opções que os municípios brasileiros realmente terão condições de aplicar na prática. $\mathrm{Na}$ Tabela 1 é possível observar os cenários criados para o presente estudo.

Tabela 1 - Cenários de soluções integradas para o gerenciamento dos RSU.

\begin{tabular}{|c|c|c|}
\hline Cenário & Descrição geral & Descrição específica \\
\hline \multirow{3}{*}{ Cenário Inercial } & \multirow{3}{*}{$\begin{array}{l}\text { Manutenção das condições atuais do sistema } \\
\text { de gestão dos resíduos sólidos urbanos do } \\
\text { município (terceirização dos serviços). }\end{array}$} & $\begin{array}{l}\text { a) Contratação de empresas terceirizadas para } \\
\text { realização da coleta, triagem, segregação, transporte, } \\
\text { destinação final e limpeza urbana. }\end{array}$ \\
\hline & & $\begin{array}{l}\text { b) Forma de coleta (Coleta seletiva atendendo } 6 \% \\
\text { da área urbana e coleta convencional no restante da } \\
\text { área urbana). }\end{array}$ \\
\hline & & $\begin{array}{l}\text { c) Disposição final em aterro sanitário situado a mais } \\
\text { de } 300 \mathrm{~km} \text { de distância do município. }\end{array}$ \\
\hline \multirow{4}{*}{ Cenário Normativo } & \multirow{4}{*}{$\begin{array}{l}\text { Criação de um novo sistema de gestão dos } \\
\text { resíduos sólidos urbanos no município de } \\
\text { acordo com a Lei 12.305/2010. Proposta } \\
\text { de instalação de uma Central Municipal de } \\
\text { Resíduos Sólidos Urbanos (CMRSU). }\end{array}$} & $\begin{array}{l}\text { a) Ampliação da coleta seletiva (coleta seletiva para } \\
\text { todos os bairros urbanos do município). }\end{array}$ \\
\hline & & $\begin{array}{l}\text { b) Implantação de programas de educação e } \\
\text { conscientização ambiental. }\end{array}$ \\
\hline & & $\begin{array}{c}\text { c) Investimentos na central de triagem e implantação } \\
\text { de uma central compostagem no município. }\end{array}$ \\
\hline & & d) Implantação de um aterro sanitário no município. \\
\hline
\end{tabular}

O cenário I (inercial) considera que não haverá investimentos no setor nos próximos 20 anos, ou seja, considera que se prossiga o método de gerenciamento aplicado no município atualmente com a terceirização dos serviços. Os custos operacionais considerados foram os custos totais com o atual gerenciamento dos resíduos. Neste cenário, há continuação da terceirização sob responsabilidade de empresas contratadas para os serviços de coleta, limpeza e manutenção dos contêineres, operação da central de triagem, transporte e disposição final dos rejeitos e limpeza urbana. Para realizar a projeção deste cenário para os próximos 20 anos foi considerado o parâmetro de aumento populacional levando em consideração o custo por habitante. Para obtenção dos dados, foi realizada uma caracterização do atual sistema aplicado e um levantamento dos custos envolvidos no atual sistema de gerenciamento dos RSU. As informações foram obtidas de forma primária, por meio de visitas técnicas e de forma secundária, por meio de informações repassadas pela SMAM de Passo Fundo/RS.

O Cenário Il é um cenário normativo, que atende à hierarquia estabelecida pela Lei 12.305 (BRASIL, 2010). Nele se propôs a ampliação da coleta seletiva para todos os bairros, implantação de programas de educação e conscientização ambiental, investimentos na consolidação de uma 
central de triagem, aproveitamento da parcela orgânica dos resíduos por meio da compostagem e a implantação de um aterro sanitário no município. Sendo assim, será proposta a instalação de uma Central Municipal de Resíduos Sólidos Urbanos (CMRSU).

Os melhores programas de reciclagem e coleta seletiva conseguem alcançar índices de 35\% do resíduo total. Para D’Almeida e Vilhena (2000), uma usina de triagem e compostagem, quando bem operada, permite diminuição de $50 \%$, em média, do volume de resíduos sólidos que seriam destinados aos aterros, permitindo, com isso, redução de custos dos serviços e aumento da vida útil dos aterros sanitários ou controlado existentes.

A Central Municipal de Resíduos Sólidos Urbanos (CMRSU) será composta por Unidade de Triagem (UT), Unidade de Compostagem (UC) e aterro sanitário. A meta proposta é reciclar por meio da triagem $20 \%$ dos RSU. Esse percentual foi definido de acordo com a realidade do município apresentada no diagnóstico (composição gravimétrica e índices de coleta) e com estudos de que esse valor pode ser atingido por meio de programas eficientes.

Além da Unidade de Triagem, a CMRSU contará com a Unidade de Compostagem, a qual tem como objetivo o aproveitamento da parcela orgânica dos RSU. Segundo Termo de Referência Técnico, elaborado pelo Ministério das Cidades (MINISTÉRIO DAS CIDADES, 2011) - Elaboração do projeto básico e executivo completo de unidade de compostagem (UCO) de fração orgânica de resíduos sólidos urbanos, as UCOs deverão ser projetadas para atender a $20 \%$ dos resíduos orgânicos, previamente segregados. A meta estabelecida para o presente cenário é que a Unidade de Compostagem aproveite $20 \%$ dos resíduos domiciliares orgânicos, além dos resíduos de roçada, poda e capina que serão totalmente aproveitados (que no diagnóstico realizado para o município objeto de estudo foi de $17 \%$ ). Portanto, a meta estabelecida para o presente cenário é que por meio da Unidade de Compostagem se aproveite $37 \%$ dos RSU. Consequentemente, a meta estabelecida com a implantação da CMRSU é reduzir a quantidade de rejeitos em $37 \%$ em relação à quantidade de RSU gerados atualmente. O balanço de massa do cenário normativo está apresentado na Fig. 3.

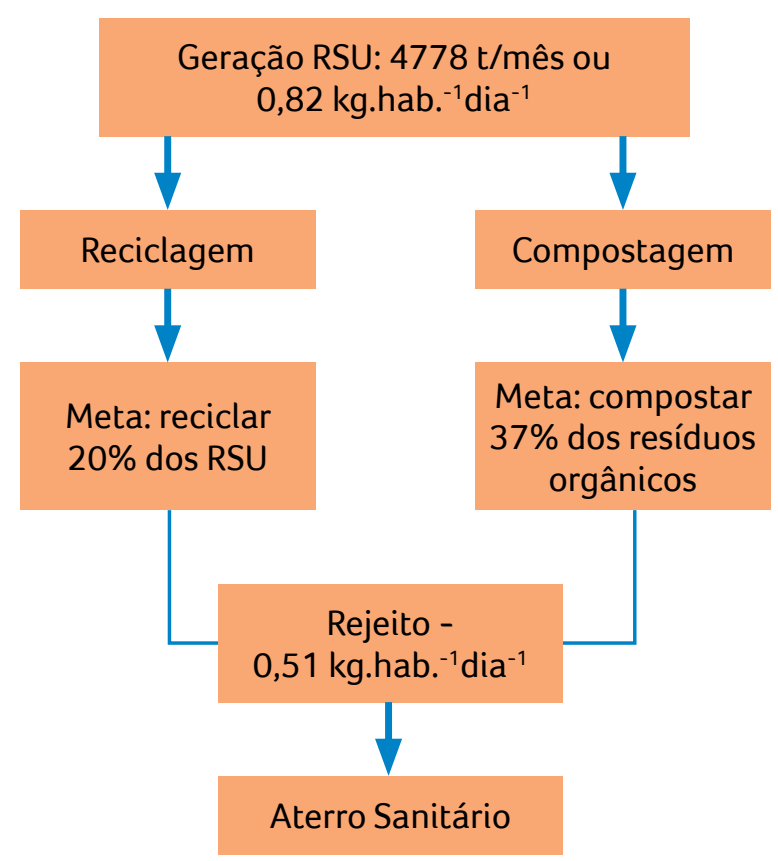

Figura 3 - Meta de aproveitamento dos RSU estabelecida para o Cenário Normativo.

O Cenário II estabelece metas para redução de rejeitos encaminhados para destinação final, por meio da ampliação da coleta seletiva e triagem dos resíduos e da reciclagem e compostagem. Estima-se reciclar 20\% dos RSU e realizar a compostagem de $37 \%$ dos resíduos sólidos orgânicos com a implementação deste. Assim, a quantidade de rejeitos se reduziria $37 \%$ em relação à quantidade de RSU gerados. A meta estabelecida para o presente cenário é atingir uma geração per capita de rejeitos de $0,51 \mathrm{~kg} \cdot \mathrm{hab} .^{-1} \mathrm{dia}^{-1}$. 
As principais caraterísticas técnicas e econômicas do Cenário II foram obtidas de uma pesquisa teórica sobre o tema com análise de pesquisas já existentes e projetos já implantados. Foi realizada também uma pesquisa de mercado, visando compilar dados de gastos com materiais e equipamentos utilizados na instalação e implantação, mão de obra especializada e serviços diversos para implementação de cada sistema. Foram realizadas consultas e pedidos de cotação a empresas do ramo, consulta a editais de licitação e planilhas de cálculos realizados em diversos municípios, consulta a trabalhos desenvolvidos sobre o tema, pesquisas no Sistema Nacional de Pesquisas de Custos e Índices da Construção Civil (SINAPI, 2015), no Sistema de Custos Rodoviários- SICRO (DNIT, 2015) e no software Pleo Planilha Eletrônica de Orçamentos, com o intuito de obter um orçamento e estabelecer um levantamento para posterior avaliação econômica.

A análise de viabilidade econômica para implantação dos cenários foi composta por um conjunto de técnicas que permitiram a comparação entre os resultados de tomada de decisões, referentes às alternativas propostas. Para que o modelo de gerenciamento dos resíduos sólidos urbanos seja implantado no município por um período de 20 anos, foi considerado:

1) Investimento inicial;

\section{2) Custos operacionais.}

A inflação não foi considerada em nenhum dos cenários, pois o índice é o mesmo, não acarretando diferenças nas comparações entre eles. Sendo assim, considerou-se que os custos aumentem de acordo com a inflação nula, pois não se leva em conta a lucratividade, visto que se trata de um serviço público.

O serviço de coleta dos resíduos sólidos domiciliares é tarifado. As taxas de coleta dos resíduos variam de acordo com o tipo da ocupação e pe- riodicidade da coleta. Esse valor é cobrado mensalmente por meio da conta de energia elétrica. Segundo informação repassada pela Secretaria Municipal da Fazenda, a arrecadação das taxas de coleta no ano de 2014 foi de $\mathrm{R} \$ 2.784 .293,10$. Existe uma lei municipal que prevê que $20 \%$ do valor arrecadado tem como destino a Secretaria do Meio Ambiente, com a finalidade de ser aplicado em atividades referentes ao gerenciamento de resíduos. É importante salientar que esse valor anual destinado para o setor não foi considerado em nenhum dos cenários.

\section{RESULTADOS E DISCUSSÃO}

O investimento inicial e os custos operacionais para os cenários foram determinados por meio do dimensionamento do mesmo para atender à demanda de geração de RSU do município. Os custos operacionais considerados foram os custos totais com o atual gerenciamento dos resíduos, conforme apresenta a Tabela 2. Neste cenário, há continuação da terceirização sob responsabilidade de empresas contratadas para os serviços de coleta, limpeza e manutenção dos contêineres, operação da central de triagem, transporte e disposição final dos rejeitos e limpeza urbana.

Tabela 2 - Custos operacionais - Cenário I

\begin{tabular}{|c|c|}
\hline Serviços & Total anual (R\$) \\
\hline Coleta & $6.594 .866,88$ \\
\hline Manutenção de contêineres & $1.637 .160,00$ \\
\hline Triagem/reciclagem & $\mathbf{7 2 . 0 0 0 , 0 0}$ \\
\hline $\begin{array}{c}\text { Operação da central de triagem, } \\
\text { transporte e disposição final }\end{array}$ & $10.070 .826,51$ \\
\hline Limpeza urbana & $3.300 .000,00$ \\
\hline Total & $\mathbf{2 1 . 6 7 4 . 8 5 3 , 3 9}$ \\
\hline
\end{tabular}

No Cenário I não há custos como investimento inicial; foram mantidos ao longo do período os custos da terceirização dos serviços, o custo total do cenário pode ser visto na Tabela 3. 
Tabela 3 - Custo total do Cenário I

\begin{tabular}{|c|c|}
\hline Ano & Custo anual (R\$) \\
\hline 1 & $21.674 .853,39$ \\
\hline 2 & $21.864 .482,40$ \\
\hline 3 & $22.055 .795,50$ \\
\hline 4 & $22.248 .792,69$ \\
\hline 5 & $22.443 .473,98$ \\
\hline 6 & $22.639 .839,36$ \\
\hline 7 & $22.824 .416,08$ \\
\hline 8 & $23.010 .340,08$ \\
\hline 9 & $23.197 .948,17$ \\
\hline 10 & $23.387 .015,81$ \\
\hline 11 & $23.577 .542,99$ \\
\hline 12 & $23.769 .754,28$ \\
\hline 13 & $23.963 .425,11$ \\
\hline 14 & $24.158 .780,03$ \\
\hline 15 & $24.355 .706,78$ \\
\hline 16 & $24.554 .205,34$ \\
\hline 17 & $24.754 .275,73$ \\
\hline 18 & $24.922 .572,87$ \\
\hline 19 & $25.092 .105,02$ \\
\hline 20 & $25.262 .759,90$ \\
\hline Total (R\$) & \\
\hline & $4698.085,48$ \\
\hline
\end{tabular}

Dessa forma, os valores utilizados para o Cenário I foram os obtidos na fase de diagnóstico do município, permanecendo a continuação da terceirização sob responsabilidade de empresas contratadas para os serviços de coleta, limpeza e manutenção dos contêineres, operação da central de triagem, transporte e disposição final dos rejeitos e limpeza urbana.

O investimento inicial para implantação do Cenário II foi orçado em $\mathrm{R} \$ 18.017 .412,43$. Um financiamento da linha Saneamento para Todos do governo federal brasileiro foi simulado para esse investimento e diluído ao longo do horizonte. Além disso, foi considerado o gasto com a aplicação da coleta seletiva, programas e educação ambiental e o gasto de operação da CMRSU, que será composto pelos gastos com insumos, tais como materiais de escritório e limpeza, contas de água, luz, internet e telefone, mão de obra, despesas com máquinas e equipamentos e despesas legais. Na Tabela 4 apresentam-se os custos operacionais para implantação do Cenário II.

Tabela 4: Custo operacional do Cenário II

\begin{tabular}{|c|c|c|c|c|c|c|c|}
\hline Ano & Coleta (R\$) & $\begin{array}{c}\text { Manutenção } \\
\text { contêineres (RS) }\end{array}$ & $\begin{array}{c}\text { Educação } \\
\text { ambiental (R\$) }\end{array}$ & $\begin{array}{c}\text { Cooperativa de } \\
\text { Recicladores (R\$) }\end{array}$ & $\begin{array}{l}\text { Operação da } \\
\text { CMRSU (RS) }\end{array}$ & $\begin{array}{l}\text { Limpeza Urbana } \\
\text { (R\$) }\end{array}$ & Total (R\$) \\
\hline 1 & $7.020 .000,00$ & $1.637 .160,00$ & $60.000,00$ & $72.000,00$ & 2.763.221,38 & $3.300 .000,00$ & $14.852 .381,38$ \\
\hline 2 & $7.080 .891,84$ & $1.651 .429,12$ & $60.370,64$ & $72.055,28$ & $2.786 .786,64$ & $3.328 .174,96$ & $14.979 .708,48$ \\
\hline 3 & $7.142 .849,28$ & $1.665 .879,04$ & $60.898,88$ & $72.685,76$ & $2.811 .170,88$ & $3.357 .296,32$ & $15.110 .780,16$ \\
\hline 4 & $7.205 .352,12$ & $1.680 .456,16$ & $61.431,77$ & $73.321,79$ & $2.835 .769,77$ & $3.386 .674,03$ & $15.243 .005,64$ \\
\hline 5 & $7.268 .400,36$ & $1.695 .160,48$ & $61.969,31$ & $73.963,37$ & $2.860 .583,31$ & $3.416 .308,09$ & $15.376 .384,92$ \\
\hline 6 & $7.331 .994,00$ & $1.709 .992,00$ & $62.511,50$ & $74.610,50$ & $2.885 .611,50$ & $3.446 .198,50$ & $15.510 .918,00$ \\
\hline 7 & $7.391 .769,84$ & $1.723 .933,12$ & $63.021,14$ & $75.218,78$ & $2.909 .137,14$ & $3.474 .294,46$ & $15.637 .374,48$ \\
\hline 8 & $7.451 .982,00$ & $1.737 .976,00$ & $63.534,50$ & $75.831,50$ & $2.932 .834,50$ & $3.502 .595,50$ & $15.764 .754,00$ \\
\hline 9 & $7.512 .739,56$ & $1.752 .146,08$ & $64.052,51$ & $76.449,77$ & $2.956 .746,51$ & $3.531 .152,89$ & $15.893 .287,32$ \\
\hline 10 & $7.573 .969,80$ & $1.766 .426,40$ & $64.574,55$ & $77.072,85$ & $2.980 .844,55$ & $3.559 .932,45$ & $16.022 .820,60$ \\
\hline 11 & $7.635 .672,72$ & $1.780 .816,96$ & $65.100,62$ & $77.700,74$ & $3.005 .128,62$ & $3.588 .934,18$ & $16.153 .353,84$ \\
\hline 12 & $7.697 .921,04$ & $1.795 .334,72$ & $65.631,34$ & $78.334,18$ & $3.029 .627,34$ & $3.618 .192,26$ & $16.285 .040,88$ \\
\hline 13 & $7.760 .642,04$ & $1.809 .962,72$ & $66.166,09$ & $78.972,43$ & $3.054 .312,09$ & $3.647 .672,51$ & $16.417 .727,88$ \\
\hline 14 & $7.823 .908,44$ & $1.824 .717,92$ & $66.705,49$ & $79.616,23$ & $3.079 .211,49$ & $3.677 .409,11$ & $16.551 .568,68$ \\
\hline 15 & $7.887 .683,88$ & $1.839 .591,84$ & $67.249,23$ & $80.265,21$ & $3.104 .311,23$ & $3.707 .384,97$ & $16.686 .486,36$ \\
\hline 16 & $7.951 .968,36$ & $1.854 .584,48$ & $67.797,31$ & $80.919,37$ & $3.129 .611,31$ & $3.737 .600,09$ & $16.822 .480,92$ \\
\hline 17 & $8.016 .761,88$ & $1.869 .695,84$ & $68.349,73$ & $81.578,71$ & $3.155 .111,73$ & $3.768 .054,47$ & $16.959 .552,36$ \\
\hline 18 & $8.071 .265,52$ & $1.882 .407,36$ & $68.814,42$ & $82.133,34$ & $3.176 .562,42$ & $3.793 .672,38$ & $17.074 .855,44$ \\
\hline 19 & $8.126 .169,12$ & $1.895 .212,16$ & $69.282,52$ & $82.692,04$ & $3.198 .170,52$ & $3.819 .478,28$ & $17.191 .004,64$ \\
\hline 20 & $8.181 .436,32$ & $1.908 .101,76$ & $69.753,72$ & $83.254,44$ & $3.219 .921,72$ & $3.845 .455,08$ & $17.307 .923,04$ \\
\hline Total & 152.133.378,12 & $35.480 .984,16$ & $1.297 .215,27$ & $1.548 .676,29$ & $59.874 .674,65$ & $71.506 .480,53$ & $321.841 .409,02$ \\
\hline
\end{tabular}


Sendo assim, para que o modelo de gerenciamento dos resíduos sólidos do Cenário Normativo seja implantado no município por um período de 20 anos, foi considerado: a) Investimento inicial com a implantação da CMRSU; b) Custos operacionais com coleta, manutenção de contêineres, educação ambiental, apoio à cooperativa de recicladores, operação da CMRSU e limpeza urbana. A Tabela 5 apresenta um quadro resumo com o custo total do Cenário II.

Tabela 5: Gasto total do Cenário II

\begin{tabular}{|c|c|c|c|}
\hline Ano & Financiamento - investimento inicial (R\$) & Custos operacionais (R\$) & Total (R\$) \\
\hline 0 & $900.870,62$ & - & $900.870,62$ \\
\hline 1 & 2.681.591,55 & $14.852 .381,38$ & $17.533 .972,93$ \\
\hline 2 & $2.578 .892,30$ & $14.979 .708,48$ & $17.558 .600,78$ \\
\hline 3 & $2.476 .193,05$ & $15.110 .780,16$ & $17.586 .973,21$ \\
\hline 4 & $2.373 .493,80$ & $15.243 .005,64$ & $17.616 .499,44$ \\
\hline 5 & $2.270 .794,55$ & $15.376 .384,92$ & $17.647 .179,47$ \\
\hline 6 & $2.168 .095,30$ & $15.510 .918,00$ & $17.679 .013,30$ \\
\hline 7 & $2.065 .396,04$ & 15.637.374,48 & $17.702 .770,52$ \\
\hline 8 & $1.962 .696,79$ & $15.764 .754,00$ & $17.727 .450,79$ \\
\hline 9 & $1.859 .997,54$ & $15.893 .287,32$ & 17.753.284,86 \\
\hline 10 & 1.757.298,29 & $16.022 .820,60$ & $17.780 .118,89$ \\
\hline 11 & $1.654 .599,04$ & $16.153 .353,84$ & $17.807 .952,88$ \\
\hline 12 & $1.551 .899,79$ & $16.285 .040,88$ & $17.836 .940,67$ \\
\hline 13 & $1.449 .200,54$ & $16.417 .727,88$ & $17.866 .928,42$ \\
\hline 14 & $1.346 .501,29$ & $16.551 .568,68$ & $17.898 .069,97$ \\
\hline 15 & $1.243 .802,04$ & $16.686 .486,36$ & $17.930 .288,40$ \\
\hline 16 & - & $16.822 .480,92$ & $16.822 .480,92$ \\
\hline 17 & - & $16.959 .552,36$ & $16.959 .552,36$ \\
\hline 18 & - & $17.074 .855,44$ & $17.074 .855,44$ \\
\hline 19 & - & $17.191 .004,64$ & $17.191 .004,64$ \\
\hline 20 & - & $17.307 .923,04$ & $17.307 .923,04$ \\
\hline \multicolumn{3}{|r|}{ Total (R\$) } & $352.182 .731,55$ \\
\hline
\end{tabular}

Na Tabela 6 está apresentado um resumo dos cenários, onde foi realizada uma média dos custos.

Tabela 6: Diferença média entre os cenários

\begin{tabular}{|c|c|c|c|}
\hline Cenário & Custo total (R\$) & Média do custo anual (R\$) & Média do custo mensal (R\$) \\
\hline Cenário I & $469.758 .085,48$ & $23.487 .904,27$ & $1.957 .325,36$ \\
\hline Cenário II & $352.182 .731,55$ & $17.609 .136,58$ & $1.467 .428,05$ \\
\hline
\end{tabular}

A análise dos cenários demonstrou que o Cenário Il é o mais viável do ponto de vista econômico, reduzindo os custos com o gerenciamento dos RSU em aproximadamente $25 \%$. Além disso, trará também uma série de outros benefícios sociais e ambientais para o município.

Um estudo semelhante realizado por Tan et al. (2014) em Iskandar, Malásia, também indicou um modelo de otimização que previu melhorar a combinação de tecnologias de tratamento de resíduos, gerando um custo otimizado em uma solução eficaz para a gestão de RSU. A alocação ótima do sistema de gerenciamento em termos de porcentagem foi aterro sanitário com recuperação de energia (14\%), incineração com aproveitamento energético (3\%), instalações de reciclagem de materiais (56\%) e compostagem (27\%). Esse cenário seria capaz de atingir a meta de energia 
renovável, atingir o objetivo de reciclagem e promover a compostagem como alternativa de redução de resíduos para a região em estudo.

Sendo assim, é possível relacionar o presente estudo com os resultados de Tan et al. (2014), pois o gerenciamento de RSU é um assunto amplo e multidimensional onde a realidade de cada região e/ou país é bem diferente, sendo necessário estudar e diagnosticar a situação do objeto de estudo para ter condições de propor melhorias no sistema.

A despesa total com o manejo dos RSU pode ser calculada por habitante. Levando-se os custos dos cenários para valores atuais, tem-se a um valor per capita médio, conforme apresentado na Fig. 4.

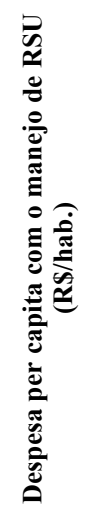

112,30

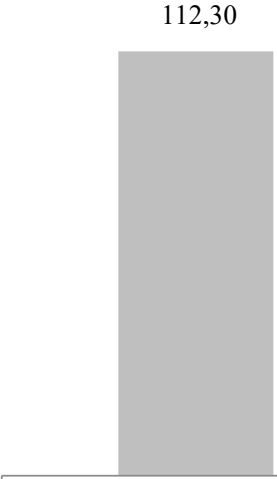

Cenário I

82,98

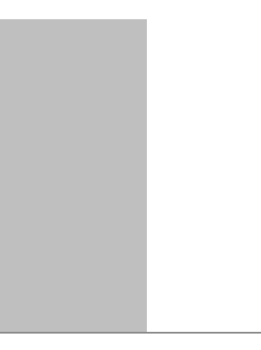

Cenário II

Figura 4 - Custo per capita com o gerenciamento dos RSU para os cenários analisados.

Segundo Diagnóstico do Manejo de Resíduos Sólidos Urbanos - 2013 (MINISTÉRIO DAS CIDADES, 2013), dentre os municípios que participaram da edição, a despesa total com o manejo dos RSU resulta em um valor médio anual de $\mathrm{R} \$ 105,74$ por habitante, partindo de um patamar de $\mathrm{R} \$ 77,98$ por habitante nas regiões Norte e Sul e chegando ao patamar de $\mathrm{R} \$ 115,99$ por habitante nas regiões Sudeste e Centro-Oeste.

Visto de outra forma, segundo o porte populacional dos municípios brasileiros de médio porte, para os municípios onde o número de habitantes varia de 100.001 a 250.000 , a despesa per capita anual com o manejo dos RSU é de $\mathrm{R} \$ 76,43$ por habitante (MINISTÉRIO DAS CIDADES, 2013).
Observa-se que o cenário I tem uma despesa per capita ( $\mathrm{R} \$ 112,30$ hab/ano) com o manejo de RSU próximo da média nacional (R\$105,74 hab/ano). Porém, quando se avalia que o município está inserido na região Sul do Brasil e o número de habitantes é de um município de médio porte, o valor do Cenário I é superior, considerando os dados apresentados no Diagnóstico do Manejo de Resíduos Sólidos Urbanos - 2013 (MINISTÉRIO DAS CIDADES, 2013).

Analisando os dados a partir do percentual que cada etapa do gerenciamento, pode-se verificar quanto cada etapa representa no valor total do custo, conforme pode ser visto na Fig. 5. 


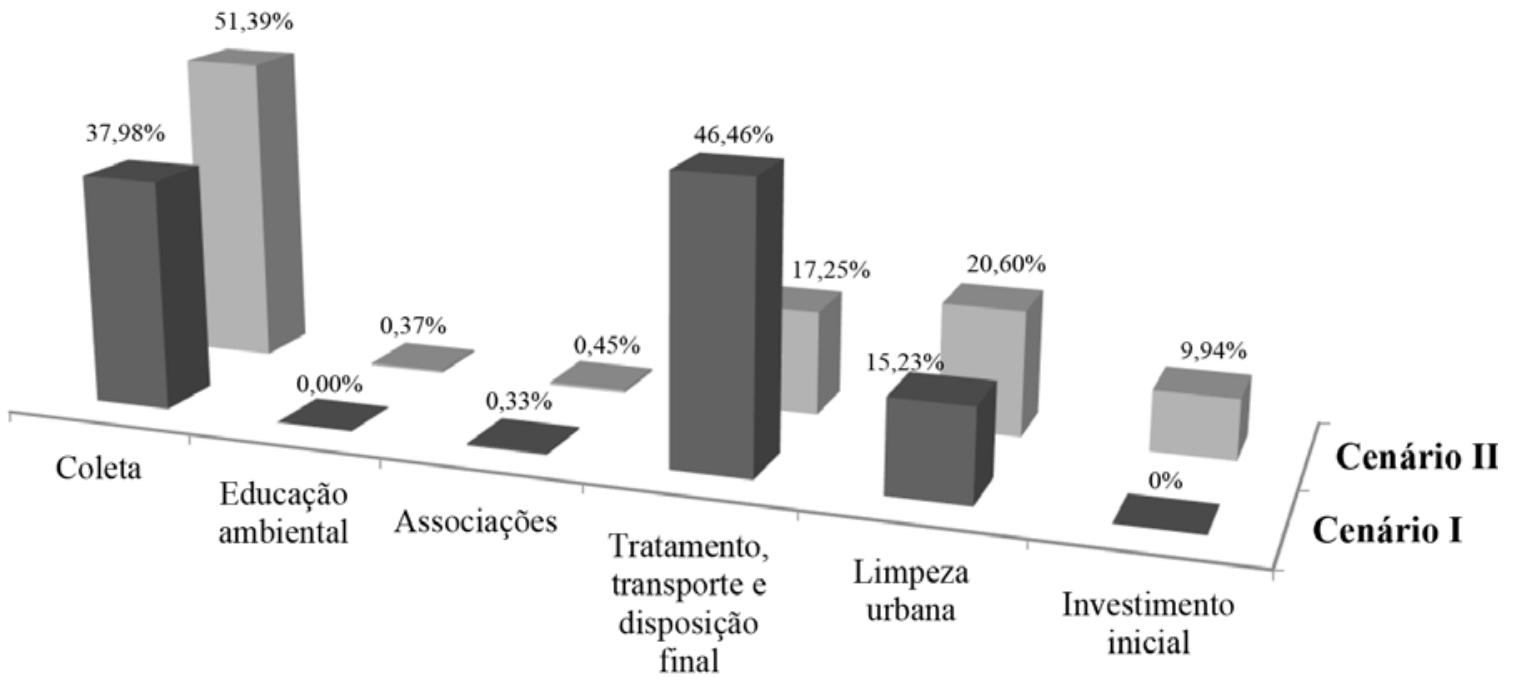

Figura 5 - Percentual de cada etapa para os cenários.

Verifica-se que o investimento inicial no Cenário II representa menos de $10 \%$ do valor anual com os custos do gerenciamento de RSU, quando distribuídos ao longo do período analisado. Sendo assim, o investimento inicial do Cenário II é recuperado pelo aproveitamento dos materiais por meio: do aumento da taxa de reciclagem, da ampliação da coleta seletiva; da triagem mais eficiente e com a compostagem, diminuindo desta forma a quantidade de rejeitos gerados.

No que se refere aos custos de triagem, transporte e disposição final, verifica-se que no Cenário I representam $46,77 \%$ do custo total; já no Cenário II os custos com investimento inicial e operação da CMRSU representam $27,19 \%$ do custo total.

Os custos diminuem significativamente no Cenário II, quando se propõe o fim da terceirização dos serviços em algumas etapas do gerenciamento. No Cenário II, propõe-se que a operação da CMRSU seja realizada pela administração municipal, proprietária da usina, e contabilizada nos custos operacionais da CMRSU, reduzindo o custo dessa etapa.
A etapa de coleta é a mais representativa nos custos de gerenciamento de RSU para os dois cenários. Isso também se confirma em outros estudos realizados, como por Lohri, Camenzind e Zurbrügg (2014) e por Guerrero, Maas e Hogland (2013). Segundo Tavares et al. (2009), a etapa de coleta pode representar até $70 \%$ dos custos totais de gerenciamento dos RSU. Esse custo poderá ser diminuído por meio de diferentes medidas que poderão ser adotadas, como tamanho e uso eficiente dos veículos de coleta, aquisição de veículos que consomem menos combustível, rotas de coleta mais eficientes, etc.

Com a implantação da coleta seletiva em todo o município, o custo do gerenciamento aumentou. Sendo assim, a eficiência na etapa de triagem e reciclagem foi melhor, diminuindo, dessa forma, a quantidade de rejeitos para disposição final, e, consequentemente reduzindo os custos totais e com os impactos ambientais globais. As vantagens de investir no gerenciamento dos RSU são inúmeras, e é imprescindível que os municípios tenham atitudes proativas buscando alternativas locais para otimizar o sistema e reduzir os custos. 
Isso também foi concluído em um estudo realizado em Pequim, na China. Os autores analisaram a otimização da ecoeficiência para o gerenciamento de RSU considerando os impactos ambientais e os custos com a gestão. Foram analisadas as etapas de coleta seletiva, compostagem, aterro sanitário e incineração. A relação de separação durante a coleta de RSU e a proporção das técnicas utilizadas para o tratamento foram os principais indicadores para medidas de otimização. Para a cidade de Pequim, um aumento na proporção de separação de resíduos durante a coleta provou ser a medida mais eficaz, com maior pontuação de ecoeficiência (YANG; ZHOU; XU, 2015).

Ferreira et al. (2014) avaliaram a taxa de retorno econômica e ambiental alcançada com a implementação de um sistema de reciclagem de resíduos em Portugal. A conclusão do estudo foi que as operações de eliminação dos resíduos provaram ser mais caras (principalmente do ponto de vista ambiental) do que a reciclagem, que reduz os custos econômicos e também os danos ambientais. Ou seja, a pesquisa se mostrou favorável à reciclagem dos resíduos e não à incineração direta. Os autores estão de acordo com a nossa opinião de que a triagem e a reciclagem são etapas fundamentais no gerenciamento do RSU quando se refere ao retorno econômico e ambiental.

No Ásia, Othman et al. (2013) analisaram a aplicação da Análise do Ciclo de Vida para a avaliação do gerenciamento de RSU para vários países asiáticos. $O$ estudo incidiu sobre a avaliação de impactos ambientais de diversas tecnologias de tratamento de resíduos e concluiu que a reciclagem, a digestão anaeróbia e os tratamentos térmicos são tecnologias eficazes para esses países. Portanto, fica claro que independentemente da região analisada, promover a reciclagem é essencial e viável, independentemente da tecnologia adotada para disposição final.
Comparando os dois cenários do presente estudo, é possível verificar que o Cenário II é o que apresenta o menor custo total. Além disso, é um Cenário que atende à Política Nacional de Resíduos Sólidos, cumprindo a Lei 12.305 (BRASIL, 2010).

Os benefícios com a implantação do Cenário II vão além dos econômicos e administrativos; compreendem também as esferas sociais e ambientais. Além da geração de emprego e renda, o município passaria a ser o responsável pela disposição final dos rejeitos gerados em seu território, eliminando-se os riscos ambientais de transportar rejeitos por mais de $300 \mathrm{~km}$ de distância, conforme vem sendo realizado.

Um dos fatores mais controversos durante a tomada de decisão acerca da adoção de uma tecnologia de tratamento e disposição final se refere às oportunidades de geração de emprego perdidas ou oportunizadas (FADE, 2014). O Cenário Il é o mais intensivo no quesito necessidade de mão de obra local, contribuindo dessa forma com a inclusão social, por empregar uma comunidade de trabalhadores pouco qualificados, compostos por catadores de materiais recicláveis, associados ou não.

Com a conscientização da população sobre educação ambiental e coleta seletiva, espera-se que ocorra uma correta segregação dos resíduos. Com uma estrutura completa de triagem, seria possível inserir e formalizar a atividade dos catadores e recicladores, valorizando-os. A reciclagem aumentaria de forma gradual, e a parcela orgânica seria aproveitada por meio da compostagem.

A compostagem apresentou várias vantagens no Cenário II. Além da possibilidade de geração de um composto orgânico de qualidade, que poderá ser utilizado para diversos fins, há redução de rejeitos encaminhados para disposição final, diminuindo os impactos ambientais decorrentes. A unidade de compostagem geraria emprego e renda, sendo possível inserção social, e os 
custos operacionais e de implantação seriam baixos, quando comparados com os custos de disposição no aterro sanitário. Yang, Zhou e Xu (2015) também chegaram à conclusão de que a compostagem é mais eficaz quando comparada a outras tecnologias de tratamento, pois gera menor impacto ambiental e os custos econômicos são menores.

No Cenário II, o volume de rejeitos enviado para disposição final seria menor, e por meio de técnicas de engenharia haveria uma solução segura de disposição, um aterro sanitário. Além disso, o Cenário II poderá ser complementado com a instalação de sistemas de aproveitamento energético, visando à comercialização de energia elétrica e a geração de créditos de carbono.

Sendo assim, verifica-se que a aplicação das diretrizes estabelecidas pela Política Nacional de Resíduos Sólidos reduz os custos com o gerenciamento dos RSU. Dessa forma, os municípios podem atender à legislação e ainda ter benefícios econômicos, ambientais e sociais.

Em muitos municípios brasileiros, o cenário inercial se repete, e as administrações públicas adotam medidas corretivas, atingidas em curto prazo, que solucionam parcialmente e momentaneamente os problemas relacionados aos RSU. Contudo, é necessário que os gestores públicos mudem essa visão, passando a adotar medidas preventivas de médio e longo prazo, pois somente dessa forma os problemas existentes serão solucionados e novos problemas serão evitados.

A CMRSU é um modelo que otimiza o sistema, reduz os custos com a gestão e preconiza acima de tudo o atendimento da Política Nacional de Resíduos Sólidos, podendo ser aplicada em outros municípios brasileiros e com condições semelhantes no mundo. A partir desse princípio, os municípios aderem a uma gestão integrada e visam ao cumprimento da hierarquia proposta para a gestão dos resíduos: não geração, redução, reutilização, reciclagem, tratamento e disposição final ambientalmente adequada dos rejeitos.

Com a implantação do Cenário Normativo, podese firmar o marco norteador para a consecução de uma gestão sustentável dos resíduos. E tal gestão ocorrerá mediante o desenvolvimento e o incentivo constantes da prática da coleta seletiva, das ferramentas relacionadas ao incremento da responsabilidade compartilhada pelo ciclo de vida dos produtos, do ordenamento das ações de reciclagem e reaproveitamento de materiais reutilizáveis e recicláveis, dos processos de monitoramento e fiscalização, da integração e educação ambiental e social e da cooperação técnica financeira entre o setor público. Além disso, outro aspecto futuro que pode ser adicionado ao cenário normativo é o aproveitamento energético no aterro sanitário.

A GIRSU é uma questão multidimensional. Muitos municípios procuram equipamentos e tecnologias inovadoras como um caminho para encontrar soluções à diversidade de problemas que enfrentam. Um estudo realizado por Guerrero, Maas e Hogland (2013) mostra que um sistema eficaz não se baseia apenas em soluções tecnológicas, mas também em aspectos que devem estar presentes para que o sistema global funcione, como a questão jurídica, institucional, ambiental, sócio cultural e econômica.

Dessa forma, fica evidente que os dados obtidos no presente estudo confirmam o relato de Guerrero, Maas e Hogland (2013). O Brasil possui uma legislação muito ampla e completa no que tange o quesito resíduos sólidos. A Política Nacional de Resíduos Sólidos considera todos os aspectos que devem estar presentes na gestão, porém falta colocar em prática os critérios estabelecidos e mudar a realidade dos municípios brasileiros.

Os serviços de gerenciamento de resíduos sólidos urbanos têm um custo bastante significativo no orçamento público, e as despesas não são 
recuperadas. Nesse sentido, é imprescindível que haja uma infraestrutura adequada, com recursos, equipamentos, manutenções, operação e equipe técnica qualificada. Dessa forma, com o interesse dos dirigentes municipais na questão de gestão de resíduos, com a participação dos usuários dos serviços e a boa administração dos recursos, é possível criar um sistema mais sustentável.

\section{CONCLUSÃO}

O presente trabalhou avaliou a viabilidade econômica de cenários voltados para o gerenciamento dos resíduos sólidos urbanos de um município Brasileiro de médio porte, verificando redução dos custos e atendimento à legislação. Conclui-se que o cenário normativo é uma alternativa efetiva para a otimização do gerenciamento dos RSU nos municípios brasileiros. Os benefícios com a implantação do Cenário II vão além dos econômicos e administrativos, compreendendo também as esferas sociais, ambientais e legais.

Os principais aspectos levantados nesta pesquisa, que podem ser aplicados a outros municípios de médio porte, estão descritos a seguir.

A coleta seletiva é uma medida de otimização no gerenciamento de RSU. Os custos dessa etapa são significativos em municípios de médio porte, entretanto esse aumento se torna irrelevante quando o aperfeiçoamento das etapas de triagem e compostagem foi atingido. Além disso, é fundamental que os municípios destinem recursos para a educação ambiental.

Com um processo de triagem eficiente e aproveitamento dos resíduos orgânicos por meio da compostagem, é possível reduzir os custos com a destinação dos rejeitos, gerar emprego e renda e diminuir os impactos ambientais.

A terceirização dos serviços pode ser uma boa alternativa em algumas etapas do gerenciamento, porém os custos operacionais são redu- zidos quando a administração é realizada pelo próprio município.

A criação de uma Central Municipal de Resíduos Sólidos Urbanos - CMRSU é uma opção viável e uma maneira de reduzir os custos com a gestão dos resíduos, além de apresentar uma série de benefícios sociais e ambientais.

O estudo realizado visou estabelecer uma nova dinâmica nas políticas municipais destinadas aos resíduos sólidos, propondo uma atuação integrada entre os serviços prestados à população, visando maior eficiência, eficácia, integração social e sustentabilidade. Com isso será possível garantir a correta gestão dos RSU de acordo com a Política Nacional de Resíduos Sólidos - PNRS, diminuindo custos e proporcionando à população melhorias contínuas no setor e, consequentemente, na qualidade do Saneamento Básico dos municípios.

É fato que a PNRS considera todos os aspectos que devem estar presentes na gestão de RSU, porém ainda falta colocar em prática os critérios estabelecidos na legislação para mudar a realidade dos municípios brasileiros. Acredita-se que estudos como esse possam diminuir a resistência dos municípios quanto à mudança, pois os resultados demonstram viabilidade. Entretanto, nessa fase de tomada de decisão, o simples gerenciamento de RSU passa a ser complexo dentro do sistema de gestão, que envolve, entre outros, conceitos políticos e culturais.

\section{AGRADECIMENTOS}

Fundação de Amparo à Pesquisa do Estado do Rio Grande do Sul - Fapergs pela concessão da bolsa para realização desta pesquisa.

\section{CONTRIBUIÇÃO DOS AUTORES}

Todos os autores contribuíram de forma igualitária. 


\section{REFERÊNCIAS}

ABDRABO, M. A. Assessment of economic viability of solid waste service provision in small settlements in developing countries: case study Rosetta, Egypt. Waste Management, v. 28, p. 25032511, 2008. https://doi.org/10.1016/j.wasman.2007.10.017

ASASE, M.; YANFUL, E.K.; MENSAH, M.; STANFORD, J.; AMPON$\mathrm{SAH}, \mathrm{S}$. Comparison of municipal solid waste management systems in Canada and Ghana: a case study of the cities of London, Ontario, and Kumasi, Ghana. Journal of Waste Management, v. 29, p. 2779-2786, 2009. https://doi.org/10.1016/j.wasman.2009.06.019

BARTONE, C.; BERNSTEIN, J.; WRIGHT, F. Investments in solid waste management. Infrastructure and Urban Development Department, The World Bank, Washington, 1990.

BRASIL. Lei n 12.305 de 2 de Agosto de 2010. Institui a Política Nacional de Resíduos Sólidos, altera a Lei n 9.605, de 12 de fevereiro de 1998 e dá outras providências. Diário Oficial da República Federativa do Brasil, Brasília, DF, 2010.

BUARQQUE, S. C. Metodologia e técnicas de contrução de cenários globais e regionais. Ministério do Planejamento Orçamento e Gestão e Instituto de Pesquisa Econômica Aplicada. Brasília, 2003.

BURNLEY, S. J. A review of municipal solid waste composition in the United Kingdom. Journal of Waste Management, v. 27, n. 10, p. 1274-1285, 2007. https://doi.org/10.1016/j.wasman.2006.06.018

COINTREAU, S.; GOPALAN, P.; COAD, A. Private sector participation in municipal solid waste management: guidance pack. SKAT, St. Gallen, Switzerland, 2000.

D'ALMEIDA, M.L.O.; VILHENA, A. Lixo Municipal: manual de gerenciamento integrado. $2^{\circ}$ ed. São Paulo: IPT/CEMPRE, 370p. 2000.

DIAZ, L. F. Book review: the science of composting by Eliot Epstein Laricaster, Technomic Publishing Co. Waste Management \& Research, v.17, n. 2, p. 66, 1999. https://doi.org/10.1177/0734242X9901700111

DNIT. Departamento Nacional de Infraestrutura e Transporte. Sistema de custos rodoviários - SICRO. Ministério de Transportes. Brasil, 2015

FADE. Fundação Apoio ao Desenvolvimento da Universidade Federal de Pernambuco. Análise das diversas tecnologias de tratamento e disposição final de resíduos sólidos urbanos no Brasil, Europa, Estados Unidos e Japão. Jaboatão dos Guararapes, PE: Grupo de Resíduos Sólidos - UFPE, 2014.

FAMURS. Federação das Associações de Municípios do Rio Grande do Sul. Informações Municipais. Rio Grande do Sul, 2009. Disponível em: <http://www.famurs.com.br/index.php/ municipios/ informacoes-municipais>. Acesso em: 15 out. 2014.

FERREIRA, S.; CABRAL, M.; DA CRUZ, N.F.; MARQUUES, R.C. Economic and environmental impacts of the recycling system in Portu- gal. Journal of Cleaner Production, v. 79, n. 0959-6526, p. 219 -230, 2014. https://doi.org/10.1016/j.jclepro.2014.05.026

GUERRERO, L. A.; MAAS, G.; HOGLAND, W. Solid waste management challenges for cities in developing countries. Journal of Waste Management, v. 33, p. 220-232, 2013. https://doi.org/10.1016/j.wasman.2012.09.008

HANRAHAN, D.; SRIVASTAVA, S.; RAMAKRISHNA, A. Improving management of municipal solid waste in India: overview and challenges. Environment and Social Development Unit, South Asia Region, World Bank, New Delhi, India, 2006.

HOORNWEG, D.; LAM, D.; CHAUDHRY, M. Waste management in China: issues and recommendations. East Asia Infrastructure Development, The World Bank, Washington, DC, USA, 2005.

JUCÁ, J. F. T.et al. Análise das diversas tecnologias de tratamento e disposição final de resíduos sólidos urbanos no Brasil, Europa, Estados Unidos e Japão. FADE - Fundação Apoio ao Desenvolvimento da Universidade Federal de Pernambuco. Jaboatão dos Guararapes, PE: Grupo de Resíduos Sólidos - UFPE, 2013.

LOHRI, C. R.; CAMENZIND, E. J.; ZURBRÜGG, C. Financial sustainability in municipal solid waste management: costs and revenues in Bahir Dar, Ethiopia. Waste Management, v. 34, p. 542-552, 2014. https://doi.org/10.1016/j.wasman.2013.10.014

LOPES, A. A. Estudo da gestão e do gerenciamento integrado dos resíduos sólidos urbanos no município de São Carlos/SP. 2003. Dissertação (Mestrado em Ciências da Engenharia Ambiental) - Escola de Engenharia de São Carlos da Universidade de São Paulo - São Carlos, 2003.

MARSHALL, R. E.; FARAHBAKHSH, K. Systems approaches to integrated solid waste management in developing countries. Waste Management, v. 33, p. 988-1003, 2013. https://doi.org/10.1016/j.wasman.2012.12.023

McDOUGALL, F.; WHITE, P.; FRANKE, M.; HINDLE, P. Integrated solid waste management: a life cycle inventory. Oxford, UK/Malden, MA: Blackwell Sci., 2 ed., 2001.

MINGHUA, Z.; XIUMIN, F.; ROVETTA, A.; QICHANG, H.; VICENTINI, F.; BINGKAI, L.; GIUSTI, A.; YI, L. Municipal solid waste management in Pudong New Area, China. Journal of Waste Management, v. 29, p. 1227-1233, 2009. https://doi.org/10.1016/j.wasman.2008.07.016

MINISTÉRIO DO MEIO AMBIENTE. Guia para elaboração de planos de gestão dos resíduos sólidos. Brasil, 2011.

MINISTÉRIO DAS CIDADES. Secretaria Nacional de Saneamento Ambiental. Sistema Nacional de Informações sobre Saneamento: diagnóstico do manejo de resíduos sólidos urbanos. Brasília: MCIDADES.SNSA, 2013.

MINISTÉRIO DAS CIDADES. Secretaria Nacional de Saneamento Ambiental. Sistema Nacional de Informações sobre Saneamen- 
to: diagnóstico do manejo de resíduos sólidos urbanos. Brasília: MCIDADES.SNSA, 2018.

MINISTÉRIO DAS CIDADES. Termo de Referência Técnico - Elaboração do projeto básico e executivo completo de unidade de compostagem (UCO) de fração orgânica de resíduos sólidos urbanos. 2011.

MORRISSEY, A.J., BROWNE, J. Waste management models and their application to sustainable waste management. Waste Management, v. 24 (3), p. 297-308, 2004. https://doi.org/10.1016/j. wasman.2003.09.005

MRAYYAN, B.; HAMDI, M. R. Management approaches to integrated solid waste in industrialized zones in Jordan: a case of Zarqa City. Journal of Waste Management, v. 26, p. 195-205, 2006. https://doi.org/10.1016/j.wasman.2005.06.008

OTHMAN, S. N.; NOOR, Z. Z.; ABBA, A. H.; YUSUF, R. O.; HASSAN, M. A. A. Review on life cycle assessment of integrated solid waste management in some Asian countries. Journal of Cleaner Production, v. 41, p. 251-262, 2013. https://doi.org/10.1016/j.jclepro.2012.09.043

PASSO FUNDO. Plano municipal de saneamento básico de Passo Fundo-RS. Prefeitura Municipal de Passo Fundo/RS, Passo Fundo/ RS, 2014.

PARTHAN, S. R.; MILKE, M. W.; WILSON, D. C.; COCKS, J. H. Cost estimation for solid waste management in industrializing regions: precedents, problems and prospects. Waste Management, v. 32, p. 584-594, 2012. https://doi.org/10.1016/j.wasman.2011.11.004

RODRIGO, J.; CASTELLS, F. Environmental evaluation of different strategies for the management of municipal solid waste in CataIonia. Life Cycle Management. In: $1^{\text {a }}$ International Conference on Life Cycle Management. Copenhagen, Denmark, p. 305-8, 2001. Anais...

SCHEINBERG, A.; WILSON, D. C.; RODIC, L. Solid waste management in the world's cities. UN-Habitat's Third Global Report on the State of Water and Sanitation in the World's Cities. Earthscan, London, 2010.

SHEN, T. T. Industrial pollution prevention. Springer Verlag. In: Gerenciamento Ambiental, Cap. II, p. 74-6, 1995.

SCHÜBELER, P. Conceptual framework for municipal solid waste management in low income countries. Working Paper, n. 9, Urban Management and Infrastructure, UNDP/UNCHS/World Bank-UMP, Nairobi, Kenya, 1996. Publicação disponível em: http:// documents1.worldbank.org/curated/en/829601468315304079/ pdf/400960Municpal1 teOframework01PUBLIC.pdf
SENG, B.; KANEKO, H.; HIRAYAMA, K.; KATAYAMA-HIRAYAMA, K. Municipal solid waste management in Phnom Penh, capital city of Cambodia. Waste Management \& Research, v. 29, p. 491-500, 2010.

SINAPI. Sistema Nacional de Pesquisa de Custos e Índices da Construção Civil. Instituto Brasileiro de Geografia e Estatística - IBGE, 2015. Disponível em: <http://www.ibge.gov.br/ home/estatistica/indicadores/precos/sinapi/defaultindice.shtm>. Acesso em: 30 jun. 2016.

SUJAUDDIN, M.; HUDA, M. S.; RAFIQUUL HOQUE, A. T. M. Household solid waste characteristics and management in Chittagong, Bangladesh. Journal of Waste Management, v. 28, p. 1688-1695, 2008. https://doi.org/10.1016/j.wasman.2007.06.013

TAN, S. T.; LEE, C. T.; HASHIM, H.; HO, W. S.; LIM, J. S. Optimal process network for municipal solid waste management in Iskandar Malaysia. Journal of Cleaner Production, v. 71, n. 0959-6526, p. 48 - 58, 2014. https://doi.org/10.1016/j.jclepro.2013.12.005

TAVARES, G.; ZSIGRAIOVA, Z.; SEMIAO, V.; CARVALHO, M. G. Optimization of MSW collection routes for minimum fuel consumption using 3D GIS modelling. Waste Management, v. 29, p. 1176-1185, 2009. https://doi.org/10.1016/j.wasman.2008.07.013

TCHOBANOGLOUS, G.; KREITH, F. Handbook of solid waste management. New York: McGraw-Hill, 2 ed., 2002.

UN-HABITAT. United Nations Human Settlements Programme. Solid waste management in the world's cities. London: UN Hun. Washington, DC. Settl. Programm. 2010.

WHITE P.; FRANKE, M.; HINDLE, P. Integrated solid waste management: a lifecycle inventory. New York: Chapman \& Hall, 1999.

WILSON, C. D.; RODIC, L.; SCHEINBERG, A.; VELIS, C. A.; ALABASTER, G. Comparative analysis of solid waste management in 20 cities. Waste Management, v. 30, n. 3, p. 237-254, 2012. https:// doi.org/10.1177/0734242×12437569

YANG, Z.; ZHOU, X.; XU, L. Eco-efficiency optimization for municipal solid waste management. Journal of Cleaner Production, v. 104 , p. $242-249$, 2015. https://doi.org/10.1016/j.jclepro.2014.09.091

ZHU, D.; ASNANI, P. U.; ZURBRÜGG, C.; ANAPOLSKY, S.; MANI, S. Improving municipal solid waste management in India: a sourcebook for policymakers and practitioners. The World Bank, Washington, DC, 2007.

ZURBRÜGG, C.; BECKER, B.; VOEGELI, Y. Cash flow in solid waste management. Sandec News, v. 8, p. 14-15, Eawag, Dübendorf, 2007. 